\title{
ON THE FINITE TIME BLOW-UP OF THE EULER-POISSON EQUATIONS IN $\mathbb{R}^{N *}$
}

\author{
DONGHAO $\mathrm{CHAE}^{\dagger}$ AND EITAN TADMOR $\ddagger$
}

\begin{abstract}
We prove the finite time blow-up for $C^{1}$ solutions of the attractive Euler-Poisson equations in $\mathbb{R}^{n}, n \geq 1$, with and without background state, for a large set of 'generic' initial data. We characterize this supercritical set by tracing the spectral dynamics of the deformation and vorticity tensors.
\end{abstract}

Key words. Euler-Poisson equations, finite time blow-up

AMS subject classifications. 35Q35, 35B30

\section{The Euler-Poisson equations}

We are concerned with the pressureless Euler-Poisson equations in $\mathbb{R}^{n}, n \geq 1$,

$$
\begin{aligned}
& \partial_{t} \rho+\operatorname{div}(\rho v)=0, \\
& \partial_{t}(\rho v)+\operatorname{div}(\rho u \otimes u)=-k \rho \nabla \phi, \quad-\Delta \phi=\rho-c, \\
& \left\{\begin{array}{l}
v(x, 0)=v_{0}(x), \\
\rho(x, 0)=\rho_{0}(x) .
\end{array}\right.
\end{aligned}
$$

The equations involve the unknown velocity field, $v=\left(v^{1}, \ldots, v^{n}\right)=v(x, t)$, local density $\rho=\rho(x, t) \geq 0$, potential function $\phi=\phi(x, t)$, and the two constants, $c$ and $k$. Here, $c \geq$ 0 is the constant "background" state; typical cases include the case of zero background, $c=0$, or the case of a nonzero background given by the average mass, $c=\bar{\rho}$, where

$$
\bar{\rho}:=\int \rho(x, t) d x=\int \rho_{0}(x) d x .
$$

Finally, $k$ is a scaled physical constant which signifies whether the underlying forcing is attractive, when $k<0$, or repulsive, when $k>0$.

The hyperbolic-elliptic system (1.1) appears in a variety of different applications, from small scale models in charge transport and plasma collision, e.g., $[18,8]$, to large scale dynamics of (clusters of) stars in cosmological waves, and expansion of the cold ions, e.g., $[1,7]$.

For the questions of local regularity and global existence of weak solutions, we refer to $[13,14,5]$ for local existence in the small $H^{s}$-neighborhood of a steady state, and to $[16,9]$ for the relaxation limit of the weak entropy solution in the isentropic and isothermal cases. Global existence with a "sufficient" amount of damping relaxation

*Received: June 18, 2008; accepted (in revised version): July 4, 2008. Communicated by Francois Bouchut.

$\dagger$ Department of Mathematics, University of Chicago, Chicago, IL 60637, USA and Sungkyunkwan University, Suwon 440-746, Korea (chae@uchicago.edu).

${ }^{\ddagger}$ Department of Mathematics, Center for Scientific Computation and Mathematical Modeling (CSCAMM) and Institute for Physical Science and Technology (IPST), University of Maryland, College Park, MD 20742 (tadmor@cscamm.umd.edu). http://www.cscamm.umd.edu/ ${ }^{\sim}$ tadmor. 
can be found in $[22,23,12]$. For the model without damping relaxation, global existence was obtained by Guo [6], assuming the flow is irrotational and the data is in the small $\mathrm{H}^{2}$-neighborhood of a constant state.

We focus our attention on the questions of global regularity versus finite-time breakdown of solutions for (1.1). In this case, the precise configuration of the initial data and the type of attractive vs. repulsive forcing play a decisive role. On the one hand, there are various non-existence results. A finite time breakdown result in the case of attractive forces, $k<0$, was proved by Makino and Perthame ([15]) for the spherically symmetric Euler-Poisson equations with pressure, subject to compactly supported $\rho(x, t)$ in $\mathbb{R}^{3}$. For the repulsive case, with similar geometry, the blow-up was deduced in [20] provided that the initial data is sufficiently large. The study of singularity formation in the model with diffusion and relaxation can be found in [24]. Local conditions for the finite-time loss of smoothness in the one-dimensional case with and without pressure were given in $[3,4]$. On the other hand, there are various results on the long time existence of strong solutions. Global regularity results for a large class of initial data near a steady state is obtained in [6]. In [19] the stability type of result was obtained with inclusion of the pressure.

All these results leave open the question of global regularity of solutions to (1.1) subject to more general settings of initial configurations, which are not necessarily confined to a "sufficiently small" neighborhood of any prefered state (including infinity). It is in this sense that we are concerned here with the global regularity vs. finite-time breakdown of solutions to (1.1). The main difficulty lies with the nonlocal nature of the forcing term, $-k \nabla \phi$, which resembles the notorious pressure term in the $3 \mathrm{D}$ incompressible Euler equations. This feature was emphasized in [11], and was the main motivation for studying the so-called 'restricted Euler-Poisson' model, where the nonlocal forcing term is replaced by a local one. It is shown that in the repulsive case, the restricted model admits global smooth solutions for a large set of initial configurations, so called sub-critical conditions which are not necessarily confined to any prefered small neighborhood. This type of 'critical threshold phenomena' was studied in $[3,10,11,2]$ via spectral dynamics, and we will use it below to derive finite time breakdown in the attractive case.

Our aim in this paper is to show that finite time blow-up is generic for the attractive Euler-Poisson equations under suitable conditions for a large class of initial data. In section 2 we show how to bypass the difficulty of nonlocal forcing by tracing the spectral dynamics of the symmetric part of the velocity gradient matrix (deformation tensor) and observing that the vanishing property of the skew-symmetric part (vorticity tensor) is preserved along the particle trajectories. In contrast to this generic finite time blow-up in the attractive case, one expects global regularity for large sets of initial configurations in the repulsive Euler-Poisson equations. These are the sub-critical initial data identified in the one-dimensional case $[3,21]$ and the two-dimensional case of the restricted Euler-Poisson equations [11]. The regularity of the non-restricted repulsive Euler-Poisson equations in $n>1$ dimensions remains an outstanding open problem.

\section{Finite time blow-up in the attractive case}

We focus on the case $k<0$, which represents the case of attractive Poisson forcing. Let us define the rescaled vorticity matrix $\Omega=\left(\Omega_{i j}\right)$ for the $n$ dimensional vector field $v=\left(v^{1}, \cdots, v^{n}\right)$ as $\Omega_{i j}:=\frac{1}{2}\left(\partial_{i} v^{j}-\partial_{j} v^{i}\right), i, j=1, \cdots, n$ (the extra $\frac{1}{2}$ factor will simplify our formulae below). In the 1 -D case we set $\Omega \equiv 0$. 
Theorem 2.1. Consider the n-dimensional Euler-Poisson equations (1.1). Assume that the initial data $\left(\rho_{0}, v_{0}\right)$ satisfies,

$$
\mathcal{S}:=\left\{a \in \mathbb{R}^{n} \mid \rho_{0}(a)>0, \Omega_{0}(a)=0, \operatorname{div} v_{0}(a)+\sqrt{-n k c}<0\right\} \neq \emptyset .
$$

Then the $C^{1}$-regularity of local classical solution with initial data $v_{0}, \rho_{0}$ cannot persist for arbitrarily long times, namely, there exists $t_{c}<\infty$ such that $\operatorname{div} v(\cdot, t) \downarrow-\infty$ as $t \uparrow t_{c}$.

REMARK 2.1. We refer to $\mathcal{S}$ as the set of supercritical configurations. We observe that in the zero-background case, $c=0$, Theorem 2.1 implies a finite time breakdown for generic non-vacuum, supercritical initial configurations where $\Omega_{0}(a)=0$ and $\operatorname{div} v_{0}(a)<$ 0 . In particular, in the $1-\mathrm{D}$ case and in the $\mathrm{n}-\mathrm{D}$ spherically symmetric case, the requirement for vanishing vorticity, $\Omega_{0}(a)=0$, is redundant.

REMARK 2.2. The finite time blow-up is a local phenomenon and does not depend on the specific domain; the same results hold for a bounded domain with a smooth boundary or a periodic domain.

REMARK 2.3. The finite-time breakdown will occur at a critical time, $t_{c}<\infty$, which satisfies

$$
t_{c} \leq \inf _{a \in \mathcal{S}} t_{c}(a)<\infty, \quad t_{c}(a):=\left\{\frac{1}{2 \sqrt{-n k c}} \ln \frac{\operatorname{div} v_{0}(a)-\sqrt{-n k c}}{\operatorname{div} v_{0}(a)+\sqrt{-n k c}}\right\} .
$$

Proof. Away from vacuum, where $\rho>0$, the momentum equation (1.1b) can be rewritten solely in terms of the velocity field,

$$
\partial_{t} v+(v \cdot \nabla) v=-k \nabla \phi, \quad\left\{(x, t) \in \mathbb{R}^{n} \times[0, \infty) \mid \rho(x, t)>0\right\} .
$$

Taking partial derivatives of (2.1) we obtain the Ricatti matrix equation,

$$
\partial_{t} V+(v \cdot \nabla) V+V^{2}=-k \Phi,
$$

where $V:=\left(\partial_{i} v^{j}\right)$ is the stress tensor and $\Phi:=\left(\partial_{i} \partial_{j} \phi\right)$ is the Hessian of $\phi$. The symmetric and the skew-symmetric parts of (2.2) satisfy

$$
\frac{D}{D t} \mathcal{D}=-\mathcal{D}^{2}-\Omega^{2}-k \Phi,
$$

and

$$
\frac{D}{D t} \Omega=-\mathcal{D} \Omega-\Omega \mathcal{D}
$$

Here, $\mathcal{D}:=\frac{1}{2}\left(V+V^{\top}\right), \Omega:=\frac{1}{2}\left(V-V^{\top}\right)$, and $\frac{D}{D t}=\partial_{t}+(v \cdot \nabla)$ amounts to path differentiation

$$
\frac{D}{D t}[\cdot](x, t)=\frac{d}{d t}[\cdot](X(a, t), t),
$$

along particle trajectories $\left\{X(a, t) \mid X_{t}(a, t)=v(X(a, t), t), X(a, 0)=a\right\}$, where $v(x, t)$ is a classical solution of the system (1.1).

Consider a particle trajectory starting at $a \in \mathcal{S}$ and let $\lambda$ be an eigenvalue of $\mathcal{D}$ associated with a normalized eigenvector $r_{\lambda}$. We are interested in the dynamics of the 
eigenvalues along such particle trajectories, $\lambda=\lambda(X(a, t), t)$. Now, the initial state at $a \in \mathcal{S}$ is assumed to be a non-vacuum state. Hence, as long as $\rho(\cdot, t)$ remains positive, (2.3a) applies, and the spectral dynamics of $\lambda$ is governed by, e.g., [11, Sec. 3]

$$
\frac{D}{D t} \lambda=-\lambda^{2}-\left\langle\Omega^{2} r_{\lambda}, r_{\lambda}\right\rangle-k\left\langle\Phi r_{\lambda}, r_{\lambda}\right\rangle
$$

Since the initial vorticity vanishes at $\mathcal{S}$, equation $(2.3 \mathrm{~b})$ tells us that $\Omega$ remains zero along the corresponding trajectory, $\Omega_{0}(a)=0 \mapsto \Omega(X(a, t), t)=0$. It follows from (2.4) that as long as the particle trajectory does not cross into a vacuum state, then

$$
\frac{D}{D t} \sum_{\lambda} \lambda=-\sum_{\lambda} \lambda^{2}-k \sum_{\lambda}\left\langle\Phi r_{\lambda}, r_{\lambda}\right\rangle, \quad \lambda \equiv \lambda(\mathcal{D}) .
$$

We observe that $\sum \lambda=\operatorname{tr}(\mathcal{D})=\operatorname{div} v$ and hence

$$
\sum \lambda^{2} \geq \frac{1}{n}\left(\sum \lambda\right)^{2}=\frac{1}{n}(\operatorname{div} v)^{2}
$$

also,

$$
\sum_{\lambda}\left\langle\Phi r_{\lambda}, r_{\lambda}\right\rangle=\operatorname{tr} \Phi=\Delta \phi=-(\rho-c) .
$$

Since $k \rho \leq 0$, we end up with the Ricatti-type inequality

$$
\frac{D}{D t} \operatorname{div} v \leq-\frac{1}{n}(\operatorname{div} v)^{2}-k c .
$$

The last inequality implies that the divergence is non-increasing along $\{X(a, t), a \in \mathcal{S}\}^{1}$

$$
\operatorname{div} v(\cdot, t) \leq M_{0}:=\sup _{a \in \mathcal{S}}\left\{\operatorname{div} v_{0}(a)\right\} \leq-\sqrt{-n k c},
$$

and hence, by (1.1a), a particle path initiated in $a \in \mathcal{S}$ remains non-vacuum for all time

$$
\frac{D}{D t} \rho=-\operatorname{div} v \cdot \rho \mapsto \rho(X(a, t), t) \geq e^{-M_{0} t} \rho_{0}(a)>0 .
$$

Moreover, if we wait long enough, solutions of (2.6) subject to $\operatorname{div} v_{0}(a)<-\sqrt{-n k c}$ will blow up as $\lim _{t \uparrow t_{c}(a)} \operatorname{div} v=-\infty$. Thus, there is a finite time breakdown on or before $t_{c}(a)$ whenever $a \in \mathcal{S}$.

Acknowledgments. This research was done while the D. Chae was visiting University of Nice and he would like to thank to Y. Brenier for his hospitality and for stimulating discussions with useful suggestions. This research of D. Chae was supported partially by KRF Grant(MOEHRD, Basic Research Promotion Fund). The research of E. Tadmor was supported in part by NSF grants DMS07-07949 and FRG07-57227, and ONR grant N00014-91-J-1076.

\footnotetext{
${ }^{1}$ In fact, $\operatorname{div} v$ remains uniformly upper-bounded for all non-vacuum paths, $\operatorname{div} v(\cdot, t) \leq$ $\sup _{\left\{a: \rho_{0}(a)>0\right\}}\left\{\operatorname{div} v_{0}(a), \sqrt{-n k c}\right\}$.
} 


\section{REFERENCES}

[1] U. Brauer, A. Rendal and O. Reula, The cosmic no-hair theorem and the non-linear stability of homogeneous Newtonian cosmological models, Class. Quantum Grav., 11, 2283-2296, 1994.

[2] B. Cheng and E. Tadmor, Long time existence of smooth solutions for the rapidly rotating shallow-water and Euler equations, SIAM J. Math. Anal., 39(5), 1668-1685, 2008.

[3] S. Engelberg, Formation of singularities in the Euler and Euler-Poisson equations, Phys. D, 98(1), 67-74, 1996.

[4] S. Engelberg, H. Liu and E. Tadmor, Critical thresholds in Euler-Poisson equations, Indiana Univ. Math. J., 50, Special Issue, 109-157, 2001.

[5] P. Gamblin, Solution reguliere temps petit pour l'equation d'Euler-Poisson, Comm. Par. Diff. Eqs., 18, 731-745, 1993.

[6] Y. Guo, Smooth irrotational flows in the large to the Euler-Poisson system in $\mathbf{R}^{3+1}$, Comm. Math. Phys., 195(2), 249-265, 1998.

[7] D. Holm, S.F. Johnson and K.E. Lonngren, Expansion of a cold ion cloud, Appl. Phys. Lett., $38,1981,519-521$.

[8] J.D. Jackson, Classical Electrodynamics, 2nd ed., Wiley, New York, 1975.

[9] S. Junca and M. Rascle, Relaxation of the isothermal Euler-Poisson system to the driftdiffusion equations, Quart. Appl. Math., 58, 511-521, 2000.

[10] H. Liu and E. Tadmor, Spectral dynamics of the velocity gradient field in restricted flows, Comm. Math. Phys., 228(3), 435-466, 2002.

[11] H. Liu and E. Tadmor, Critical thresholds in $2 D$ restricted Euler-Poisson equations, SIAM J. Appl. Math., 63, 1889-1910, 2003.

[12] T. Luo, R. Natalini and Z. Xin, Large time behavior of the solutions to a hydrodynamic model for semiconductors, SIAM J. Appl. Math., 59, 810-830, 1999.

[13] T. Makino, On a local existence theorem for the evolution of gaseous stars, Patterns and Waves (eds. T. Nishida, M. Mimura and H. Fujii), North-Holland/Kinokuniya, 459-479, 1986.

[14] T. Makino and S. Ukai, Sur l'existence des solutions locales de l'quation d'Euler-Poisson pour l'volution d'toiles gazeuses, J. Math. Kyoto Univ., 27, 387-399, 1987.

[15] T. Makino and B. Perthame, Sur les solutions à symétrie sphérique de l'éuation d'EulerPoisson pour l'évolution d'étoiles gazeuses, Japan J. Appl. Math., 7, 165-170, 1990.

[16] P. Marcati and R. Natalini, Weak solutions to a hydrodynamic model for semiconductors and relaxation to the drift-diffusion equation, Arch. Rat. Mech. Anal., 129, 129-145, 1995.

[17] P.A. Markowich, A non-isentropic Euler-Poisson model for a collisionless plasma, Math. Meth. Appl. Sci., 16, 409-442, 1993.

[18] P.A. Markowich, C. Ringhofer and C. Schmeiser, Semiconductor Equations, Springer, Berlin, Heidelberg, New York, 1990.

[19] T. Luo and J. Smoller, Rotating fluids with self-gravitation in bounded domains, Arch. Rat. Mech. Anal., 173, 345-377, 2004.

[20] B. Perthame, Nonexistence of global solutions to Euler-Poisson equations for repulsive forces, Japan J. Appl. Math., 7(2), 363-367, 1990.

[21] E. Tadmor and D. Wei, On the global regularity of sub-critical Euler-Poisson equations with pressure, J. European Math. Society, 10, 757-769, 2008.

[22] D. Wang, Global solutions and relaxation limits of Euler-Poisson equations, Z. Angew. Math. Phys., to appear.

[23] D. Wang, Global solutions to the equations of viscous gas flows, Pro. Royal Soc. Edinburgh: Section A., to appear.

[24] D. Wang and G.Q. Chen, Formation of singularities in compressible Euler-Poisson fluids with heat diffusion and damping relaxation, J. Diff. Eqs., 144, 44-65, 1998. 